Meta

Journal des traducteurs

Translators' Journal

\title{
Restrictions paradigmatiques et traduction de schémas d'arguments
}

\section{Pierre Lerat}

Volume 53, numéro 2, juin 2008

La traduction des séquences figées

The Translation of Frozen Sequences

URI : https://id.erudit.org/iderudit/018528ar

DOI : https://doi.org/10.7202/018528ar

Aller au sommaire du numéro

Éditeur(s)

Les Presses de l'Université de Montréal

ISSN

0026-0452 (imprimé)

1492-1421 (numérique)

Découvrir la revue

Citer cet article

Lerat, P. (2008). Restrictions paradigmatiques et traduction de schémas d'arguments. Meta, 53(2), 434-442. https://doi.org/10.7202/018528ar
Résumé de l'article

Les schémas d'arguments sont sujets à des restrictions. Les expressions prédicatives et leurs compléments ne se combinent pas librement de classe à classe. Les noms d'actions, et aussi les noms d'agents, peuvent " condenser " des schémas en rendant implicites leurs compléments. Deux concepts sont opératoires pour décrire les restrictions paradigmatiques au niveau du schéma d'arguments : collocation conceptuelle (variation sémantique) et paradigme phraséologique (variation stylistique). Les conséquences pour la traduction sont observées sur des corpus textuels (juridiques et vinicoles) et sur le Web.
Ce document est protégé par la loi sur le droit d'auteur. L'utilisation des services d'Érudit (y compris la reproduction) est assujettie à sa politique d'utilisation que vous pouvez consulter en ligne.

https://apropos.erudit.org/fr/usagers/politique-dutilisation/ 


\title{
Restrictions paradigmatiques et traduction de schémas d'arguments
}

\author{
PIERRE LERAT \\ Université Paris XIII, Paris, France \\ pierre.lerat@wanadoo.fr
}

\begin{abstract}
RÉSUMÉ
Les schémas d'arguments sont sujets à des restrictions. Les expressions prédicatives et leurs compléments ne se combinent pas librement de classe à classe. Les noms d'actions, et aussi les noms d'agents, peuvent «condenser» des schémas en rendant implicites leurs compléments. Deux concepts sont opératoires pour décrire les restrictions paradigmatiques au niveau du schéma d'arguments: collocation conceptuelle (variation sémantique) et paradigme phraséologique (variation stylistique). Les conséquences pour la traduction sont observées sur des corpus textuels (juridiques et vinicoles) et sur le Web.
\end{abstract}

\begin{abstract}
Schemes of arguments are subject to restrictions. Predicative expressions and their complements do not freely combine with each other from one class to another. Nouns of actions, and also nouns of agents, can "condensate" schemes by making their complements implicit. Two concepts are operative to describe paradigmatic restrictions as far as the scheme of arguments is concerned, i.e., conceptual collocation (semantic variation) and phraseologic paradigm (stylistic variation). Consequences on translation are observed in textual corpora (in law and winemaking) and on the Web.
\end{abstract}

\section{MOTS-CLÉS/KEYWORDS}

paradigme, schéma d'arguments, collocation conceptuelle, phraséologie

\section{Introduction}

En dehors des énoncés proverbiaux, qui eux aussi sont polylexicaux et soumis à des restrictions distributionnelles et transformationnelles internes en même temps qu'à une interprétation non compositionnelle (cf. Mejri 1997), le figement lexical est avant tout une affaire de catégories grammaticales: expressions nominales, verbales, adjectivales, déterminatives, adverbiales, prépositionnelles et conjonctives en français (cf. Gross 1996). Il est important d'inventorier langue par langue, et selon leur système propre en matière de parties du discours, toutes les séquences figées, car elles constituent une part importante des difficultés de traduction.

Une autre part importante est faite d'idiotismes en matière d'enchaînements non figés entre noms et verbes, noms et adjectifs, verbes et adverbes, etc. C'est ce dont essaient de rendre compte des concepts comme "phraséologie» et «collocation». La phraséologie prend en compte les expressions plus ou moins consacrées dans lesquelles entre un mot; les collocations partent elles aussi d'un mot pour rationaliser les cooccurrents typiques. Dans les deux cas, on risque d'être prisonnier d'une linguistique du mot, dont les limites sont désormais bien connues. 
Une façon de dépasser ces limites est la prise en compte de schémas d'arguments. Ce niveau d'analyse est propre à expliciter la syntagmatique des expressions prédicatives et de leurs compléments au sens large, sujets compris. Par-delà les compatibilités d'«hyperclasses», qui sont des "catégories sémantiques générales» comme HUMAIN (Guenthner 1998: 49), il est possible de mettre en évidence, notamment dans les langues spécialisées, un jeu limité et précis de «classes d'objets» et de "prédicats appropriés». Toutefois, on ne peut pas appliquer à tous les éléments d'une classe d'objets toutes les expressions prédicatives qui vaudraient pour un élément donné de la classe; ainsi, en français juridique de France, on ne promulgue pas tout texte juridique normatif, mais seulement les lois.

Il y aurait probablement plus d'inconvénients que d'avantages à rendre polysémique l'idée de figement lexical, qui est claire dans la théorie et dans la pratique de Mejri et de Gross, pour l'appliquer à de telles contraintes. En parlant de «figement paradigmatique» (Lerat 2003), j'ai pris le risque d'introduire inutilement du flou. Comme mon souci est de faire avancer la connaissance de la variabilité des schémas d'arguments mémorisés globalement, par exemple une formulation comme promulgation d'un décret ministériel au J. O., il vaut mieux parler de «restrictions», terme général qui s’applique sans inconvénients à l'usage réel face à la combinatoire générale, qui est surgénérative. Comme on dit aussi, par exemple, parution d'une circulaire ministérielle au $B$. O., on peut observer une marge de manœuvre langagière et en même temps des restrictions: un paradigme restreint d'expressions prédicatives appropriées (publication et parution, pas édition), un autre de lieux-supports (J. O., $B$. O., rien que des organes officiels), un autre d'autorités-auteurs (ministérielle, municipale, rien que des lieux de pouvoir administratif).

Ainsi, les restrictions paradigmatiques prises en compte portent soit sur le centre d'un schéma d'arguments (une expression prédicative), soit sur sa périphérie (à gauche ou à droite). C'est à ce niveau que se situe une partie importante des difficultés de la traduction, comme on va le voir dans des exemples empruntés à une thématique abstraite (le droit) et à une thématique concrète (la vinification).

\section{Figement lexical et paradigme}

La notion de «degré de figement» (Gross 1996: 154) ne relativise nullement le figement lexical mais au contraire en indique clairement les modalités et les limites: ainsi, rater le coche est globalement une expression verbale figée parce que, d'une part, syntaxiquement sa polylexicalité ne l'empêche pas d'interdire toute manipulation distributionnelle (sur le déterminant) ou transformationnelle (telle que la mise au passif) et, d'autre part, son sens n'est pas compositionnel: il s'agit de ne pas savoir profiter d'une occasion qui se présente, et non pas d'un moyen de transport de l'ancien temps. Le fait que l'on puisse dire également louper le coche ou manquer le coche montre seulement qu'il y a trois expressions verbales figées en concurrence synonymique, avec en position initiale une "possibilité de paradigme» (Gross 1996: 16).

Un tel paradigme est interne à l'expression figée. Au demeurant, il y a ouverture de paradigme uniquement en cas de défigement interne, et les enchaînements au sein d'un schéma d'arguments ne sont pas en cause. Les classes d'objets constituent des paradigmes, mais elles entretiennent avec leurs prédicats appropriés des relations qui 
ne sont pas de l'ordre du figement mais de l'affinité: «Chaque prédicat est défini [...] par son domaine d'arguments qui lui est propre [...]. Nous n'appelons pas figement cette restriction du domaine des arguments de certains verbes par rapport à d'autres » (Gross 1996: 6).

L'expérience des langues spécialisées, où pourtant la prévisibilité des enchaînements est plus grande que dans les usages ordinaires, montre qu'à partir d'un paradigme d'objets, celui des <prérogatives>, et d'un "paradigme de prédicats appropriés » (Lerat 2003: 124) tel que "acquérir + être déchu de + jouir de + se prévaloir de», on ne peut pas amalgamer automatiquement tout membre de l'un et tout membre de l'autre. Les langues seraient relativement simples si l'on pouvait combiner "des éléments fixes (invariants) et une ou plusieurs positions ouvertes (variables)» (Le Pesant et Mathieu-Colas 1998: 26). En fait, il n'y a pas d'invariants. Si l'on prend être déchu de, on ne peut l'appliquer à droit de grève, et réciproquement. Ce ne sont pas seulement les mots qui sont matière à paradigmes, mais les schémas d'arguments euxmêmes, du fait des conceptualisations dont ils résultent (le droit de grève est inaliénable dans la législation française).

\section{Les restrictions paradigmatiques dans les schémas d'arguments}

Si l'on entend par schéma d'arguments une «prédication atomique formée d'un prédicat et de ses arguments typés» (Guenthner 1998: 46), à cette définition logique correspond dans une langue naturelle un «schéma de phrase» (Guenthner 1998: 46) ou «schéma d'énoncé» (Lerat 2002b) qui se lexicalise et s'actualise selon le système de chaque langue. Il existe des parallélismes à peu près parfaits entre prédicats nominaux, tels que fr. demandeur d'asile (22400 occurrences sous Google au singulier, 114000 au pluriel) // de. Asylbewerber (56 000) et Asylsuchender (2370) // en. asylum seeker (213 000 au singulier, 1230000 au pluriel), ou encore fr. demandeur d'emploi (98 600 au singulier, 187000 au pluriel) // de. Stellensuchender (7380), Stellenbewerber (3060), Arbeitsuchender (2270) et Arbeitssuchender (2080) // en. job seeker (1 510000 au singulier, 3580000 au pluriel).

L'allemand propose deux conceptualisations: celui qui se déclare candidat à un emploi et celui qui en cherche un, deux entités distinctes pour les économistes; la distinction est faite en français pour les mêmes raisons, qui tiennent à l'existence d'une ontologie socioéconomique partagée: on parle aussi dans cette langue de recherche d'emploi (1 040 000) et de chercheur d'emploi (141000).

Il existe en principe des équivalents parfaits, puisque décidés comme tels dans une législation bilingue (comme au Canada), ou trilingue (comme en Suisse). Ils ne sont pas pour autant grammaticalement parallèles, comme on le voit avec devoir de vie commune et obligation to live together au Québec (K: 508). L'autorité du législateur détermine les dénominations à retenir, mais la forme des dénominations est ellemême dépendante de la «stylistique comparée».

Bien plus, au sein de la même langue, l'usage réel n'autorise pas toutes les possibilités ouvertes par la morphologie et la syntaxe. Ainsi, dans beaucoup d'emplois, pension et retraite sont suffisamment substituables l'un à l'autre pour que l'on puisse considérer comme synonymes les schémas d'arguments «N0 humain est retraité» et "N0 humain est pensionné» (en Belgique, sinon en France). Il reste que être en retraite n'a rien à voir avec être en pension (Lerat 2002b: 70). 
Il existe aussi de grandes différences statistiques entre les formulations verbales et nominales. Ainsi, demandeur d'asile (22 400) est plus courant que demander l'asile + demander un asile + demander asile (respectivement $7890+136+2690$ ), du fait de l'importance du statut dans le droit international. Mais le plus frappant est la différence entre demandeur d'emploi (98 600) et demander un emploi (307); c'est que le français recourt surtout à des expressions avec le verbe support faire: demande d'asile (48 000) et demande d'emploi (175 000).

Enfin, d'une langue à l'autre, il existe des différences de conceptualisation qui se reflètent dans les schémas d'arguments, y compris au sein d'un même domaine de connaissances. Un exemple juridique frappant est celui de la subrogation, qui est un concept en relation avec un droit, avec une personne qui confie ce droit à une autre et avec cette autre qui l'exerce en son nom. Le français y voit une affaire entre des personnes (le subrogeant et le subrogé) là où l'allemand privilégie le droit, qui «se déplace» (Übergang, Abtretung) d'une personne à l'autre (cf. Lerat 2002b : 74). C'est au niveau global du noyau propositionnel que se situe l'équivalence.

\section{Restrictions à gauche et à droite}

Dans le cas où l'expression prédicative est nominale, le moyen de la «conjuguer» est le recours à un «verbe support», qui joue un rôle morphologique plus que sémantique. Un travail récent (Lerat 2005) sur les emplois prédicatifs de droit (comme possibilité reconnue à une personne), clairement distincts de la classe d'objets des écrits normatifs (droit des biens, droit commercial, etc.) ainsi que de la classe d'objets des taxes (droits de succession, droits d'accise, etc.), met en évidence plusieurs façons de conjuguer ce prédicat nominal:

- verbes supports d'état: avoir droit à, avoir le droit de, être en droit de;

- verbe causatif: donner droit à;

Il est clair que le bon usage de droit, entouré à la fois de son verbe support, de la préposition, du déterminant requis et de son complément soit nominal, soit verbal à l'infinitif, résulte dans une large mesure de règles locales.

Si l'on considère en effet également les restrictions à droite, on voit que l'on a «naturellement» avoir droit à N, plus difficilement avoir droit à Vinf, et inversement avoir le droit de Vinflà où avoir le droit de $N$ est nettement moins «naturel» que avoir un droit de (par exemple avoir un droit de passage). Après être en droit de, seul un infinitif est possible, après donner droit à, un nom l'est également.

Parmi les restrictions à droite, l'effacement d'un complément logiquement requis est surtout observable dans les textes spécialisés, où le lecteur est assez «initié » pour qu'une telle "condensation» (cf. Lerat 2002a: 158-160) ne soit nullement une gêne pour la communication. Hors contexte, éleveur dénomme suffisamment, dans le monde agricole, un professionnel qui vit de l'élevage des animaux. La condensation consiste ici en l'ellipse du complément typique : la brièveté du nom d'agent n'empêche pas le renvoi à la connaissance d'un contenu conceptuel élaboré, celui où «un N0 humain élève des N1 animés non humains». Dans un contexte vinicole, changement d'évidence: l'éleveur est un vinificateur, il élève un $<$ vin $>$, il se consacre à l'élevage $\mathrm{du}<$ vin $>$, et les compléments de moyen sont devenus des $<$ cuves $>$ et des $<$ fûts $>$. S'il y a eu un jour métaphore, ce qui est plus que probable, il s'agit maintenant d'une 
catachrèse, et le sens est transparent si l'on a quelque familiarité avec le domaine de connaissances concerné.

Inversement, il existe des prévisibilités très fortes à droite de certains verbes. Ainsi, après impartir, on ne peut trouver que délai, du moins dans le domaine du droit (temps imparti est attesté sur le Web, mais seulement en matière éducative et sportive).

\section{Les conceptualisations concurrentes: les paradigmes de collocations conceptuelles}

5.1. Comme beaucoup de concepts flous, utilisés trop commodément pour caractériser une «multiplicité de rapports» (Kocourek 1991: 198), collocation peut prendre un sens précis moyennant une spécification. Les travaux portant sur les collocations conceptuelles, depuis une dizaine d'années (Heid 1994: 239), montrent que les prédicats verbaux, nominaux et adjectivaux peuvent correspondre à des gammes de conceptualisations. Il s'agit dans le cas présent de «restrictions conceptuelles» (Prandi 1998: 42) qui limitent "la distribution de mots sur la base de modèles de procès et d'états de choses cohérents et cognitivement adéquats» (p. 36). Une telle approche s'applique très bien à des processus industriels, économiques ou commerciaux, mais aussi à des schémas d'arguments juridiques, comme le montre l'usage de «collocations conceptuelles» dans l'étude de la langue du droit constitutionnel (Gautier 2004: 117). De telles «restrictions conceptuelles» ne sont pas propres aux textes spécialisés, même si elles y abondent, et il peut sembler excessif d'opposer catégoriquement collocations ordinaires et «specialized lexical combinations» à la façon de L'Homme et Bertrand (2000).

5.2. Il n'est pas toujours facile non plus de distinguer ce qui est requis sémantiquement et ce qui est variante stylistique (abordé plus loin sous couvert de paradigme phraséologique). Impartir un délai (A 149) diffère-t-il vraiment de fixer un délai (A 34, A 39, A 253) ? S'il apparaît que l'un se dit plutôt de la part d'une <autorité> et l'autre indifféremment de la part d'une < autorité $>$ ou d'une $<$ partie $>$ dans une relation plus égalitaire (contractuelle), on ne sera pas tout à fait en présence du même schéma d'arguments. Pour en juger sur une base moins étroite qu'un corpus textuel donné ( $Q$, en l'occurrence), le mieux est de faire des recherches sur ces chaînes de caractères tel jour, sur le Web (sous Google, dans le cas présent). Pour fixer un délai, qui est très fréquent (8340 occurrences), dès les premières pages référencées il apparaît que les deux classes de sujets sont représentées. En revanche, accorder un délai (8810) est inégalitaire (en termes de rapport de force). Il en va de même pour impartir un délai (629), octroyer un délai (224), consentir un délai (109), assigner un délai (63) et concéder un délai (5). Ce qui est remarquable, et qui confirme la thèse selon laquelle les langues spécialisées ne sont que des emplois spécialisés des langues naturelles (cf. notamment Lerat 1995), c'est que dans toutes ces formulations chaque verbe conserve exactement sa valeur ordinaire; ainsi, consentir, c'est «faire un geste» (unilatéralement), et assigner ne peut être le fait que d'une autorité indiscutable (le Prince ou même Dieu, d'après les 63 occurrences).

5.3. Comment bien traduire ces nuances? N’étant pas spécialiste de la traduction, je postule par commodité qu'il n'y a pas moyen de faire mieux que les corédacteurs 
d'un même texte dans un pays où l'égalité des langues fait partie de la culture politique, la Suisse, ou que les responsables des différentes versions d'un traité communautaire. Mais comme je suis philologue de formation, je confronte ce que j'ai relevé dans ces textes (voir Q) et ce qu'atteste le Web, ce qui conduit à prendre également en compte déterminer un délai (196, dont A 37) et prévoir un délai (19 600, dont A 253). Voici deux exemples suisses pour impartir un délai:

- «la Feuille officielle suisse impartit un délai à des actionnaires» (S 682, avec une autorité textuelle métonymique dans le rôle d'agent): de. eine Frist setzen, it. assegnare un termine;

- «le vendeur impartit un délai à l’acheteur» (S 226h): de. eine Frist setzen, it. concedere un termine.

Le Web fournit des informations convergentes: de. eine Frist setzen (3630, dont A 34 et A 39), bien plus courant que eine Frist bestimmen (498). Pour l'italien, assegnare un termine (716) et concedere un termine (332) sont usités, mais le traité d'Amsterdam préfère fissare un termine (A 37), qui est bien attesté (6700) et surtout stabilire un termine (A 34, A 39 et A 253), qui l'est également (3250). Pour l'anglais, les gros bataillons sont du côté de set a deadline (71 900, dont A 34 et A 253), mais aussi de set a time limit (70 100, dont A 34), encore que des textes officiels recourent aussi à lay down a time limit (117, dont A 149) ou lay down a deadline (52).

L'orientation du procès met l'accent sur l'agent ou le laisse implicite, et le prédicat lui-même peut disparaître le cas échéant, comme le montre suffisamment ci-dessous la construction de fixer un délai et de ses variantes dans le traité d'Amsterdam, avec en regard les formulations retenues dans trois autres langues par des locuteurs natifs travaillant sous le contrôle de hauts responsables politiques:

fr. «Le Conseil fixe un délai» (A 39) // de. «Der Rat setzt eine Frist» // en. «the Council shall set a deadline» // it. "il Consiglio stabilisce un termine»;

fr. «délais fixés à l'article 189 B du Traité» (A 253, avec implicitation de l'agent) // de. «die in Artikel 189 b des Vertrags enthaltenen Fristen» // en. «the deadlines set out in Article 186 b of the Treaty» // it. «termini stabiliti dall'articolo 189 b del trattato (le traité est acteur)»;

fr. «délais qu'ils [les traités] impartissent» (A 149, avec un lieu devenu agent) // de. "aus den darin enthaltenen Fristen» (avec un lieu qui reste lieu) // en. «the time limits laid down by the Treaties» // it. «termini che gli stessi [trattati] prescrivono»

fr. «délais prévus par la procédure de codécision» (A 204) // de. «Fristen im Rahmen des Mitentscheidungsverfahrens» (avec une simple indication de source, non d'agent) // en. «time limits under the co-decision procedure» // it. «termini per lo svolgimento della procedura di decisione» («pour le déroulement de la procédure»: but, cette fois).

5.4. Pour prendre un domaine plus concret, on va voir ci-dessous comment est conceptualisé le schéma "N0 humain vinifie un <vin>» dans la terminologie officielle et non officielle dans quelques langues de l'Europe.

Autour du verbe: fr. vinifier (3260, nécessairement transitif direct avec pour complément un <vin>), élever un vin (29), élaborer un vin (394); de. Wein erzeugen (657, équivalent officiel dans R 1999) et Wein herstellen (1290); en. make a wine (8520); es. elaborar un vino (377).

Autour du nom d'action: fr. vinification (166 000), élevage d'un vin (52), élaboration d'un vin (399, dont R 1999); de. Weinherstellung (56 900, dont R 1999), Weinbereitung 
(85 000), Vinifikation (10 800), Weinerzeugung (10000); en. winemaking (725 000), wine-making (484 000), vinification (6000 fois the vinification, qui permet de distinguer automatiquement l'anglais du français), sans oublier une rareté: refinement of wine (37, dont R 1999); es. vinificación (17 600) et elaboración de vino (6110).

Autour du nom d'agent, devenu plus proche de l'œnologue que du vigneron: fr. vinificateur (4720), éleveur (non quantifiable pour cause de polysémie, mais soutenu par négociant-éleveur [732], du fait de l'organisation de la profession en France), élaborateur (qui lui aussi implicite son complément mais a été retenu dans R 2004); de. Weinerzeuger (16 700), Weinhersteller (8620), Weinbereiter (237); en. winemaker (393 000, dont R 2004), wine grower (13 400); es. vinificador (269), elaborador de vino (63) et même, par condensation, elaborador (R 2004). Là encore, les langues naturelles gardent tous leurs droits: le vigneron étant proche de la vigne, l'éleveur peut en être un sous-traitant lointain, l'élaborateur compte beaucoup sur le laboratoire. On peut être winemaker sans être wine grower, depuis que l'on parle de flying winemaker (1220 en un mot, 83 avec trait d'union). Comment traduire un tel choc des cultures? Peut-être par «consultant tous azimuts» (François Gorin, Télérama, 14 novembre 2004)!

\section{Les formulations concurrentes : les paradigmes phraséologiques}

Comme collocation, phraséologie n'échappe pas à une suspicion d'à-peu-près. Si l'on n'ajoute pas une précision, on ne peut que souscrire à l'opinion avertie d'une slaviste: «La phraséologie n'est rien d'autre qu'un substitut de contexte, aléatoire et non problématisé» (Slodzian 2000: 72). C'est le cas des «séries phraséologiques» de Bally (1951), qui sont une masse de faits hétérogènes, de l'avis même de l'auteur (p. 73). C'est pourquoi il vaut mieux laisser à Bally sa dénomination et en choisir une voisine mais distincte.

En proposant de prendre en compte des paradigmes phraséologiques, on met en concurrence explicite des formulations substituables les unes aux autres moyennant une variation stylistique (registre, niveau de langue, etc.). Le traducteur n'est pas en position de force pour faire prévaloir la langue ordinaire contre la langue de bois, si l'attente du commanditaire est conformiste, mais les deux listes ci-dessous, avec à gauche le schéma d'arguments en français ordinaire et à droite son synonyme en français du dimanche, montrent comment un recueil composite d'énoncés, celui de Google fin 2004 (à suivre!), reflète le rapport des forces stylistiques, tous supports numérisés confondus:

$\begin{array}{ll}\text { payer une redevance (2150) } & \text { / acquitter une redevance (356) } \\ \text { accorder un crédit (2390) } & \text { / octroyer un crédit (634) } \\ \text { renvoi du procès }(1540) & \text { / ajournement du procès (457) } \\ \text { arrêter un suspect (130) } & \text { / appréhender un suspect (86) } \\ \text { mettre sa signature (327) } & \text { / apposer sa signature (5660) } \\ \text { timbrer l'enveloppe (28) } & \text { / affranchir l'enveloppe (117) }\end{array}$

Les médias, qui sont fortement représentés sur Internet, sont rassurants dans le cas d'ajournement, qui est délaissé hors des tribunaux et des chroniques judiciaires, mais les administrateurs, qui y figurent aussi en bonne place grâce à leurs sites institutionnels, le sont moins par leur conservatisme (apposer fait juridique, donc bénéficie d'une plus-value de professionnalisme: on dit apposer les scellés / une plaque / une mention, etc.). 


\section{Conclusion}

C'est au niveau du groupe de mots que se situent beaucoup de difficultés linguistiques de la traduction. Le schéma d'arguments ne constitue pas le seul point d'ancrage important, mais c'est le plus systématisable dans l'état actuel des connaissances. Ce n'est pas un hasard si une grande partie des trouvailles d'étudiants vantées par la directrice d'une école de traduction sont des idiotismes à base de collocations conceptuelles équivalentes, comme le montre cette illustration (avec les formulations en anglais à gauche, et à droite les façons idiomatiques de traduire en français) de l'idée (juste) que «le sens est [...] conceptuellement plus vaste que la partie qui en est explicitée par l'énoncé» (Seleskovitch et Lederer 1984: 271):

"go out into the world: entrer dans la vie

establish yourselves in your chosen careers: vous aurez commencé à faire carrière set up new homes: fonder un foyer

decisions about your children's education: l'orientation scolaire de vos enfants become figures of some importance and influence: vous compterez parmi les notables".

Ce niveau d'analyse permet de se repérer en linguiste dans un océan de contextes accessibles instantanément sur Internet. Il ne s'agit pas, en effet, de simples cooccurrences brutes et aléatoires, mais de compatibilités entre expressions prédicatives et compléments au sein de schémas à la fois logiques, sémantiques et syntaxiques (cf. Lerat 2004).

En prenant des exemples dans des domaines frontaliers entre terminologie (et culture spécialisée) et langue commune (et culture générale), il a été possible de mettre en évidence le fait que l'hypothèse de dénominations terminologiques clairement distinctes se heurte à des phénomènes de culture, de typologie linguistique, de mémoire collective et de lexicalisation (officielle et, surtout, professionnelle).

\section{RÉFÉRENCES}

BALLY, Ch. (1951): Traité de stylistique française, vol. I, Paris, Klincksieck.

Gautier, L. (2004): «Terminologie et phraséologie comparées du droit constitutionnel en français et en allemand», in MejRI, S. (dir.), L'espace méditerranéen: une idiomaticité partagée, Tunis, Ceres, pp. 113-126.

Gross, G. (1996): Les expressions figées en français, Paris, Ophrys.

Guenthner, F. (1998): «Constructions, classes et domaines: concepts de base pour un dictionnaire de l'allemand» Langages 131, pp. 45-55.

Heid, U. (1994): "On the Way Words Work Together. Topics in Lexical Combinatories", in Martin, W. et al. (eds), Euralex 1994 Proceedings, Amsterdam, Vrije Universiteit, pp. 226257.

Kocourek, R. (1991): La langue française de la technique et de la science, Wiesbaden, Oscar Brandstetter.

L'homme, M.-C. et C. Bertrand (2000): "Specialized Lexical Combinations: Should they be described as Collocations or in Terms of Selectional Restrictions", Euralex 2000 Proceedings, in Heid, U. et al. (eds), Stuttgart, Universität Stuttgart, pp. 497-506.

Le Pesant, D. et M. Mathieu-Colas (1998): «Introduction aux classes d'objets», Langages 131, pp. 6-33.

LERAT, P. (1995): Les langues spécialisées, Paris, PUF.

Lerat, P. (2002a): «Vocabulaire juridique et schémas d'arguments juridiques», Meta 47-2, pp. 155-162. 
LERAT, P. (2002b) : «Un niveau d’analyse privilégié pour les langues de spécialités européennes: le schéma d'énoncé», in Schena, L. et L. T. Soliman (dir.), Prospettive linguistiche della nuova Europa, Milano, Egea, pp. 67-77.

Lerat, P. (2003): «Le figement paradigmatique», Cahiers de lexicologie 82, pp. 117-126.

Lerat, P. (2004): «Web et terminologie philologique», Linguistica Antverpiensia 3.

Lerat, P. (2005): «Le prédicat sémantique droit sur le Web», Lexique, terminologie, discours, LINX 52, Mélanges offerts à Marie-Françoise Mortureux, pp. 155-161.

MejRI, S. (1997): Le figement lexical. Descriptions linguistiques et structuration sémantique, Tunis, Publications de la Faculté des Lettres de la Manouba.

Prandi, M. (1998): «Contraintes conceptuelles sur la distribution: réflexions sur la notion de classe d'objets", Langages 131, pp. 34-44.

Seleskovitch, D. et M. Lederer (1984): Interpréter pour traduire, Paris, Didier Erudition.

SLodzian, M. (2000): «L'émergence d'une terminologie textuelle et le retour du sens» in BÉjoInt, H. et P. Thoiron (dir.), Le sens en terminologie, Lyon, Presses de l’Université, pp. $61-85$

\section{Corpus}

A : Traité d'Amsterdam (1997, fr., de., en., it.), <http://www.europa.eu.int>.

K: Kasirer, N. (1997): «What is vie commune? Qu'est-ce que living together?», in Mélanges Paul-André Crépeau, Cowansville (Québec), Yvon Blais, pp. 508-509.

Q: Quadrirédacteur (2003): fichier de 3500 entrées juridiques (et autour) en français et leurs équivalents en trois langues (de., en., it.), <http://www-lli.univ-paris13.fr/ressources/ quadri $>$.

R 1999: Règlement (CE) n 1493/1993 du Conseil du 17 mai 1999 portant organisation commune du marché vitivinicole.

R 2004: Règlement (CE) 316/2004 du 20 février 2004 («Désignation, dénomination, présentation et protection de certains produits vitivinicoles»).

S: Code des obligations / Obligationenrecht / Codice delle obbligazioni (1993), Berne, Chancellerie Fédérale de la Suisse. 